\title{
Optical Information Processing
}

\author{
Bahram Javidi \\ University of Connecticut \\ Department of Electrical and Systems Engineering \\ 260 Glenbrook Road \\ Storrs, Connecticut 06269-3157
}

This May 1992 special section of Optical Engineering is dedicated to optical implementation of information processing, pattern recognition, and neural networks. Thirteen invited papers appear in this section and cover five topics: nonlinear joint transform correlators, the application of joint transform correlators in text retrieval, optical correlation techniques and their applications, the application of optical devices in information processing, and neural networks.

Javidi, Parchekani, and Tang describe binary representation of gray-scale nonlinear joint transform correlators in the first paper. In the second paper, Hahn and Flannery present an investigation of binary joint transform correlators for a large body of diverse images. The third paper, by Kim and Berra, discusses the application of joint transform correlators in text retrieval.

In the fourth paper, Kumar et al. describe minimum squared error synthetic discriminant functions. Hassebrook, Rahmati, and Kumar discuss hybrid composite filter banks for distortion invariant pattern recognition in the fifth paper.

The sixth paper, by Javidi and Zhang, describes experiments on nonlinearly transformed matched filters. In the seventh paper, Drolet et al. present real-time implementation of binary phase-only filters using a liquid crystal television. Marshall and Benner discuss in the eighth paper the use of matched filters and holographic ring detectors for sizing opaque spherical particles.

Molley and Kast describe in the ninth paper automatic target recognition by acousto-optic devices. The tenth paper, by Kirsch et al. , investigates the potential use of an Epson liquid crystal television in optical information processing.

Casasent describes the combination of optical correlation, feature extraction, and neural networks in the eleventh paper. The twelfth paper, by Huynh, Walkup, and Krile, presents $\mathrm{a} \mathrm{BaTiO}_{3}$-based optical quadratic neural network implementing the perceptron algorithm. The final paper, by Paek, Liao, and Gharavi, describes a derivation of neural network models and their computational circuits for associative memory.

I hope that the papers presented in this special section contribute to a better understanding of some of the current research in the area of optical information processing.

I would like to thank the authors who contributed the papers and the reviewers for their comments and suggestions. I am grateful to Brian J. Thompson for giving me the opportunity to organize this special section and a special thanks must be given to the Optical Engineering editorial staff for their excellent support in its preparation.

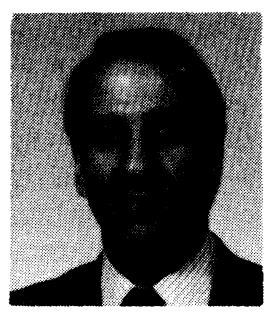

Bahram Javidi received a BS degree from George Washington University in 1980 and MS and PhD degrees from Pennsylvania State University in 1982 and 1986, respectively, all in the field of electrical engineering. He is currently an associate professor of electrical engineering at the University of Connecticut. His research interests include optical information processing, pattern recognition, digital signal processing, and communication systems. In 1990 he was named a presidential young investigator by the National Science Foundation. 